\title{
Louis Van Delft, Les moralistes. Une apologie
}

\section{Benedetta Papasogli}

\section{(2) OpenEdition}

\section{Journals}

\section{Edizione digitale}

URL: http://journals.openedition.org/studifrancesi/8243

DOI: $10.4000 /$ studifrancesi.8243

ISSN: 2421-5856

\section{Editore}

Rosenberg \& Sellier

\section{Edizione cartacea}

Data di pubblicazione: 1 mai 2009

Paginazione: 166-167

ISSN: 0039-2944

\section{Notizia bibliografica digitale}

Benedetta Papasogli, «Louis Van Delft, Les moralistes. Une apologie», Studi Francesi [Online], 157 (LIII ] I) | 2009, online dal 30 novembre 2015, consultato il 13 janvier 2021. URL: http://

journals.openedition.org/studifrancesi/8243 ; DOI: https://doi.org/10.4000/studifrancesi.8243

Questo documento è stato generato automaticamente il 13 janvier 2021.



Studi Francesi è distribuita con Licenza Creative Commons Attribuzione - Non commerciale - Non opere derivate 4.0 Internazionale. 


\title{
Louis Van Delft, Les moralistes. Une apologie
}

\author{
Benedetta Papasogli
}

\section{NOTIZIA}

LOUIS VAN DELFT, Les moralistes. Une apologie, Paris, Gallimard, «Folio Essais Inédit», 2008, pp. 461.

1 Louis Van Delft ha dedicato, come tutti sanno, l'intera sua vita di studioso a definire il moralista classico e insieme a togliere steccati, ad abolire confini per assegnare a questo scomodo personaggio territori sempre più grandi. Egli stesso ricapitola la propria opera, da La Bruyère moraliste a Les spectateurs de la vie, nella figura di un allargarsi di onde concentriche, di cui questa brillante e vivacissima apologia rappresenta certamente l'onda che va più lontano. Una apologia: il titolo è provocatorio: siamo fuori dal genere impeccabilmente accademico in cui si iscriveva per esempio, più di vent'anni or sono, lo studio fondamentale Le moraliste classique; con gli anni la penna si è fatta più giovane, la verve più libera; siamo ai limiti del pamphlet ma anche del testamento morale, dell'opera di divulgazione ma anche del saggio critico alto, nel genere di Morales du Grand Siècle di Bénichou. Il destinatario è cambiato: il lettore specialista trarrà il suo frutto, ma, soprattutto, il "nouveau venu" sarà preso per mano, e condotto a scoprire, non ordinati sentieri, bensì quanto sia complesso, magmatico e affascinante il mondo della moralistica classica.

2 Impossibile qui render conto nei dettagli di questo libro dove l'impegno di sintesi, teso all'estremo, non rinuncia tuttavia a raccogliere il maggior numero possibile di suggestioni. Ci limitiamo perciò a rilevare alcuni aspetti, o alcuni accenti di insistenza da cui il libro prendere un'aria di novità. Intanto, un uso sapiente della citazione, questa prima "forma breve", che sgorga dalla memoria di infinite letture e contribuisce al succo, al sapore, alla "substantifique moelle" di un discorso sempre eloquente, degno di una scena di teatro o di tribunale. Poi altri aspetti di metodo: prendendo come un 
tutto quel "secolo di duecent'anni" o poco più che va da Montaigne a Chamfort, e anzi operando fughe prospettiche verso l'Antichità, verso il primo umanesimo, o verso l'aforisma moderno, Van Delft smantella una storiografia legata all'ordine delle "generazioni" o all'emergere del singolo "ritratto". Si muove tra scorci rapidissimi, tra fili labirintici. L'ordine è tematico, cioè discutibile; è potentemente soggettivo, cioè riflette ragioni e passioni di una vita di ricerca. Ripartendo dal problema di definire ("ma non troppo"), l'autore risuscita la "tela di fondo", ossia il quadro epocale in cui si iscrive la stagione d'oro dei moralisti; poi, forte di una nuova e sterminata bibliografia sul tema del "theatrum mundi", adotta questa figura come accesso centrale alla problematica dei moralisti; in seguito sfrutta a fondo la categoria antropologica, nel dittico formato dal modello del "caractère" e dalla metafora dell'"anatomia morale"; affronta infine il discorso delle forme, che altri invece hanno considerato prioritario nella definizione del genere, per dire come questi "moderni" di qualche secolo fa appaiono antesignani della nostra modernità letteraria.

Due tratti mi sembrano particolarmente validi in questa apologia scritta, non contro l'ignorante di letteratura, ma contro colui che è indifferente alla ricerca di senso, e non si pone le grandi questioni dell'esistenza. Van Delft, ricostruendo il lignaggio delle saggezze antiche, vera premessa alla moderna scrittura morale, sottolinea come forse nessun altro la densità filosofica di una inchiesta sulla condizione umana, fiorita in interrogazioni non meno radicali di quelle che il pastore leopardiano formula camminando sotto le stelle. E nelle ultime pagine del libro sono appunto le grandi filosofie del xx secolo ad apparire prive di senso a Perplexe, piccolo principe di una nuova favola, forse anche perché esse hanno tagliato i ponti con quel filone di saggezza morale che ha continuato a scorrere quietamente nel deserto della storia. Secondo tratto importante: cittadino del mondo, Van Delft ha frequentato a fondo le tradizioni morali di altri paesi oltre alla Francia. Allarga continuamente lo sguardo all'Inghilterra, all'Italia, alla Spagna, alla Germania. Getta semi di nuove ricerche: intesa così, come inquietudine esistenziale e dinamica meditativa, la saggezza dei moralisti attinge a un grande fondo comune senza cui non ci sarebbe la pittura del tempo, né potremmo ascoltarne la musica, né sapremmo recepire la "lezione di anatomia" di scienziati gravi e puri che celebrano intorno alla finitudine umana un rituale di verità. È merito dunque di questa apologia dei moralisti focalizzare l'essenziale, fosse pure oggi inattuale e desueto, e convocare intorno ad esso un immenso numero di testimoni, così che la piattaforma del Seicento francese divenga realmente un osservatorio sulle arti, le scienze, le letterature del mondo.

4 Tra gli accenti messi da Van Delft non tutti troveranno, è appena il caso di dirlo, consenso unanime. Tra le due linfe maggiori della moralistica classica, antichità stoica o epicurea e morale agostiniana, egli opta risolutamente per la prima. È un antico dibattito nel quale lo studioso tende a ridimensionare la parte di scoperta del profondo, il gioco dell'interiorizzazione della maschera, che s'innesta su una nozione biblica e agostiniana di "cuore" e che - d'altra parte - ha portato alcuni a vedere nello psicanalista odierno 1'“avatar" del moralista classico. Rinuncia, in tal modo, ad approfondire e ad articolare il rapporto del moralista con quel suo vicino di casa che è il maestro spirituale. Tra le scienze moderne la psicologia è proprio quella che gli sta meno a cuore. Da parte nostra siamo convinti, da sempre, che il rapporto fra moralistica e spiritualità sia uno dei nessi più fecondi da esplorare per introdursi nella cultura del grande secolo, e siamo grati a Van Delft che in vari suoi scritti, e anche in 
qualche pagina di quest'ultimo libro, ha mostrato di sottoscrivere questa tesi e di valorizzarla. 